\title{
Comparison of Partly Revealed Anisotropic Microstructures Using Grid Intersepts as Applied to Zirconium Tubes
}

\author{
Marin Lagacé, Lisa Rodrigue and Michel Trudeau
}

Hydro-Québec Research Institut, 1800 boul. Lionel-Boulet, Varennes, Québec, Canada, J3X 1S1

One of the challenges facing microscopists is to objectively compare microstructures. Usually, differences in grain size distribution are used. For anisotropic materials, grain aspect ratio and orientation must be used in conjunction with size distribution. Unfortunately, in some cases like multi-phases materials, it is difficult to completely revealed all the grains boundaries using standard etching techniques. In those cases, we cannot rely on individual grain measurements.

On example is zirconium pressure tubes holding the uranium rods used in atomic generators. These tubes are composed of two distinct zirconium phases (see Figure 1). Chemical attack reveals the two phases very well but does not reveal the grain boundaries between two alpha phase grains. The shape of these grains can be guessed from the shape of the beta phase but no grain boundary is present to objectively delimitate the grains. Therefore, traditional characterization parameters like grain size cannot be used but the average intersept length of a grid with the alpha phase of the structure can give us very useful information.

To get this value, the image is first thresholded and binarized. A grid is overlaid on the image and the intersection of the grid with the alpha phase is taken (see Figure 3). The length of each intersept is measure and tabulated. In order to get statistical results, the analysis was done on 100 different images giving from 15000 to 40000 intersepts depending on microstructure size. To take into account anisotropy, the analysis is done with rotating the grids by $5^{\circ}$ increments over $180^{\circ}$. The average intersept length is plotted on a polar graph for every angle [1].

The plots obtained for the zirconium tubes are ellipses. The orientation of the major and minor axes give the orientation of the microstructure. Their ratio is proportional to the level of anisotropy. Their values relate to grain size in as much as the bigger the average intersept length, the bigger the average grain size. Care must be taken not to mistake this value for the actual average grain size (see Figure 2) as grain shapes are diverse and cannot be easily related to the average intersept length [2]. Using this technique, it is easy to reveal similarities and differences that would not have been visible from simple visual inspection of the micrographs. For example, the results and micrographs for three different zirconium tubes are shown in Figure 4. From simple visual inspection, it would not have been possible to see that the level of anisotropy of Sample A is the same as Sample B but much different than Sample C. Furthermore, the average grain size in the vertical direction $\left(90^{\circ}\right)$ is the same (but we do not know the actual value) for Sample B and Sample C although their anisotropy level are different. So, although direct morphological parameters such as grain size are not actually measured by this technique, very useful comparative results can be extracted.

References

[1] John C. Russ, Computer-Assisted Microscopy: The Measurement and Analysis of Images, Plenum Press, New York, 1990.

[2] Erwin E. Underwood, Quantitative Stereology, Addison-Wesley, Reading, 1970.

[3] The image analysis software used was designed and written by the author.

[4] This work was sponsored by Atomic Energy Canada and Hydro-Québec Research Institute 

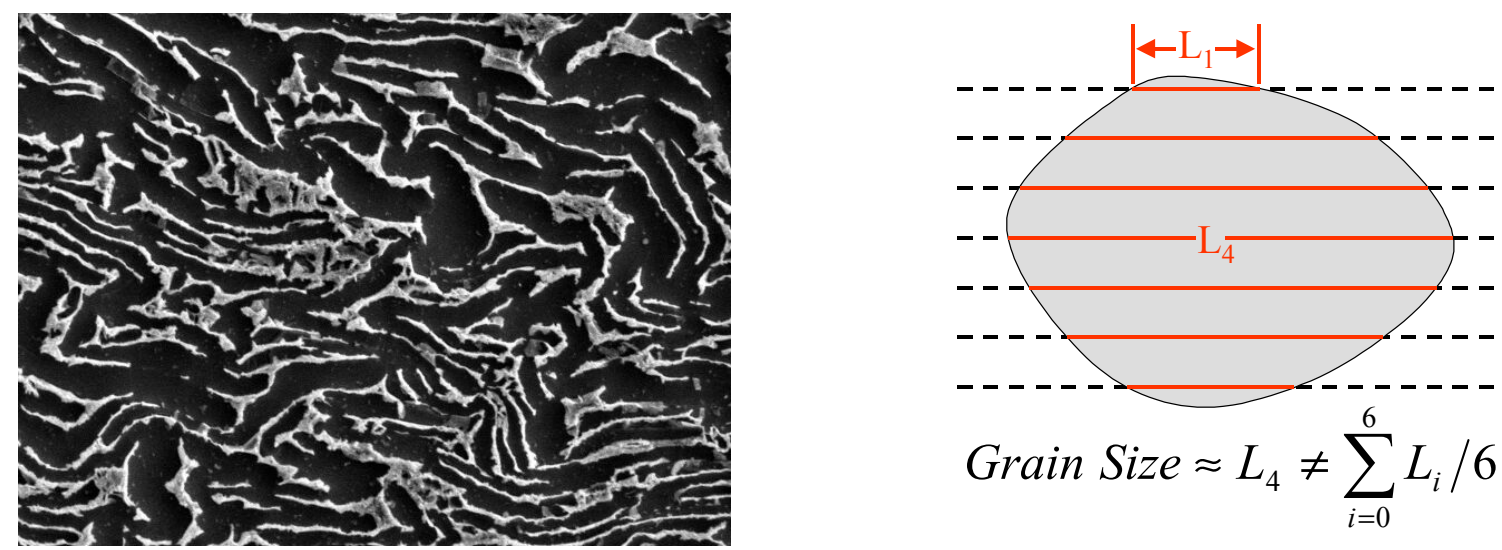

FIG1. Typical micrograph of a zirconium tube microstructure.

FIG2. Demonstration that the average intercept length is not the same as the grain size.

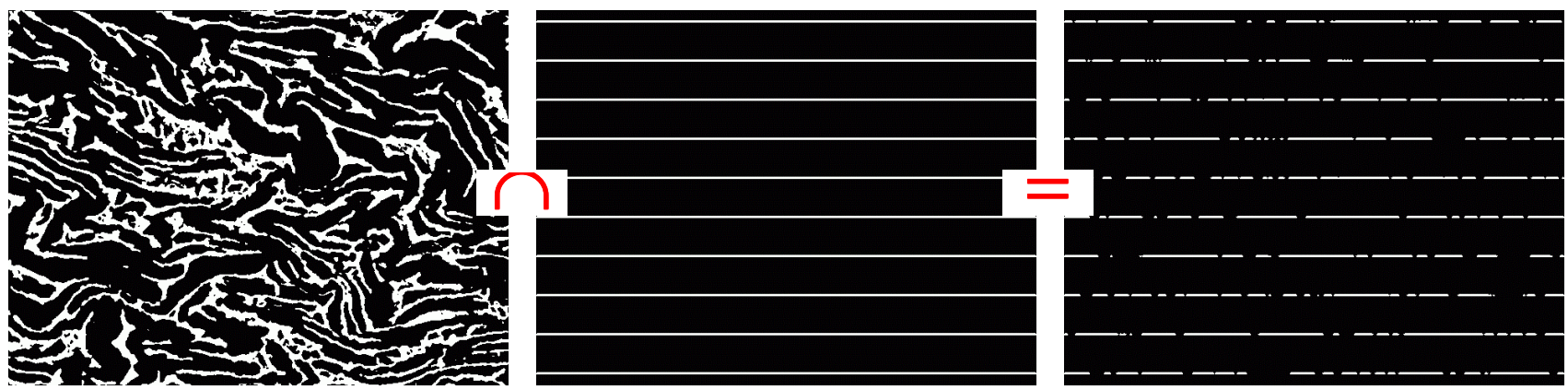

FIG 3. Intersection of a grid with the binary image of the microstructure to give the intersepts.
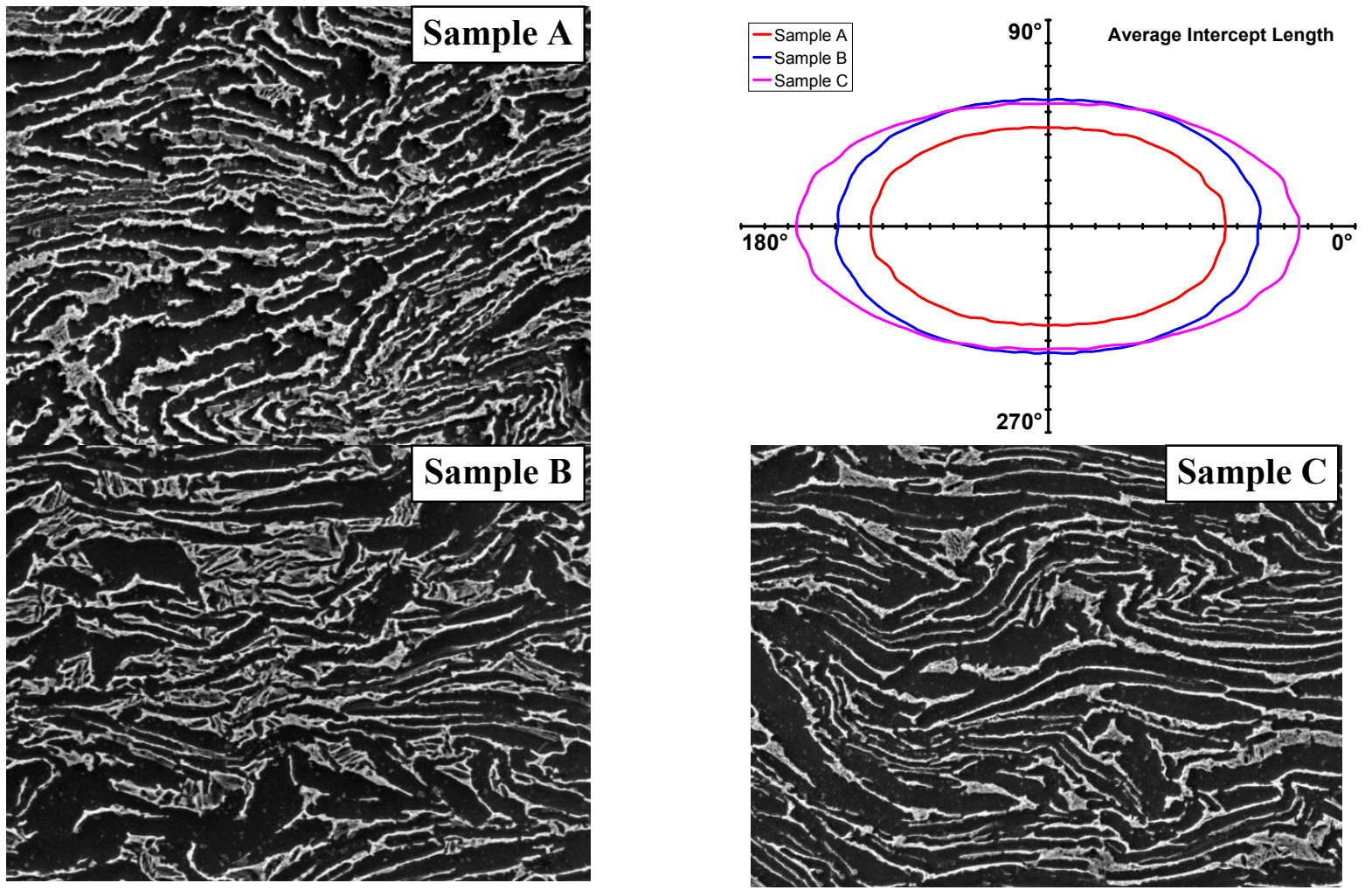

FIG 4. Comparison of three zirconium tube samples. 